\title{
Occurrence and Characteristics of Hospitalizations During First-Line Chemotherapy Among Individuals with Metastatic Colorectal Cancer
}

This article was published in the following Dove Press journal: Cancer Management and Research

\author{
Michael J Fisch (D) \\ Michael Grabner (D) ${ }^{2}$ \\ Daniel S Mytelka $\mathbb{1 D}^{3}$ \\ Amit D Raval $\mathbb{D}^{2}$ \\ Lee Bowman (iD ${ }^{3}$ \\ David M Kern $\mathbb{D D}^{2}$ \\ Collin Churchill ${ }^{3}$ \\ Joseph Singer ${ }^{2}$ \\ Stewart Wetmore ${ }^{3}$ \\ John Barron ${ }^{2}$ \\ Michael Eleff ${ }^{4}$
}

'AIM Specialty Health, Chicago, IL, USA; ${ }^{2}$ HealthCore, Inc., Wilmington, DE, USA; ${ }^{3}$ Eli Lilly and Company, Indianapolis, IN, USA; ${ }^{4}$ Anthem, Inc., Indianapolis, IN, USA

Correspondence: Michael Grabner HealthCore, Inc., I 23 Justison St, Suite 200, Wilmington, DE 1980I, USA

Tel + I 302-230-2000

Fax +I 302-230-2020

Email mgrabner@healthcore.com
Objective: Choosing chemotherapy for metastatic colorectal cancer (mCRC) requires balancing clinical effectiveness and risk of complications. This study characterized realworld inpatient/emergency department (ED) hospitalizations during first-line chemotherapy among individuals with mCRC.

Methods: This retrospective cohort study used data from medical and pharmacy claims. All patients had $\mathrm{mCRC}$ with $\geq 1$ claim for $\geq 1$ of the 5 most frequently utilized first-line chemotherapy agents (fluorouracil, oxaliplatin, bevacizumab, irinotecan, capecitabine). The main outcome was all-cause hospitalizations (inpatient or ED setting) identified from claims via ICD-9/10-CM coding from index date until 30 days after the end of first-line chemotherapy or last available data.

Results: A total of 717 individuals (mean age 55 years; $58 \%$ male; ECOG 0/1/2+/missing in 44\%/39\%/6\%/11\%; median follow-up 116 days) met study criteria. Thirty-four distinct chemotherapy regimens were used. Overall, $40 \%$ of patients had $\geq 1$ hospitalization ( $n=285$; total 415 hospitalizations); $12 \%(n=85)$ had $\geq 2$ hospitalizations. The median time to first hospitalization was 52 days; median inpatient length of stay was 4 days; infections/ neutropenia (21\%) and bowel-related complications (17\%) were the most common issues associated with inpatient hospitalizations. In univariate analyses, insurance plan type, geographical location, ECOG, and renal disease were associated with hospitalization. In multivariable analyses, ECOG $\geq 1$ was associated with a $67 \%$ increase $(p<0.01)$ in the odds of hospitalization vs $\mathrm{ECOG}=0$.

Conclusion: Approximately $40 \%$ of patients with mCRC were hospitalized during the study period. Hospital stays were typically short. Further research is needed to determine how many of these hospitalizations may be avoidable. We also observed a large amount of variation in regimens used in the first-line setting.

Keywords: colorectal cancer, chemotherapy-associated hospitalization, outcomes research, observational study, claims analysis

\section{Plain Language Summary}

Colorectal cancer (CRC) is one of the most common cancers in the United States. Hospitalizations while receiving anticancer treatments (such as chemotherapy) can reduce the effectiveness of treatment. Understanding the rates and drivers for unplanned chemotherapy-related hospitalizations is important when considering treatment choices, later lines of therapy, and clinical course.

In an analysis of administrative claims data, a cohort of 717 patients (mean age 55y; 58\% male) with metastatic CRC was identified who received at least one of the five most frequently 
used first-line chemotherapy agents for CRC (fluorouracil, oxaliplatin, bevacizumab, irinotecan, or capecitabine). A substantial proportion of patients $(40 \%)$ were hospitalized while receiving chemotherapy (hospitalization proportions by drug: 40\% 5-fluorouracil, $38 \%$ oxaliplatin, $42 \%$ bevacizumab, $49 \%$ irinotecan, $39 \%$ capecitabine). An ECOG performance status $\geq 1$ was positively associated with the occurrence of a hospitalization; no other patient demographic or clinical characteristics affected hospitalization rates. Hospital stays were typically short and associated with infections, neutropenia, or bowel-related complications.

These data provide an overview of the range of expected hospitalization rates associated with some of the first-line systemic therapies for metastatic CRC. This information may be useful during patient discussions to broadly quantify the risks of hospitalization from first-line chemotherapy and develop anticipatory intervention plans to mitigate adverse events.

\section{Introduction}

As the third most common cancer in the United States ${ }^{1}-$ and the second leading cause of cancer-related deaths ${ }^{2}-$ colorectal cancer (CRC) has a substantial impact on patients, healthcare resource utilization, and costs. Hospitalizations while receiving anticancer treatments (such as chemotherapy) can lead to dose delays or reductions that reduce the effectiveness of treatment, and may also expose patients to the risk of infection and increase financial and caregiver burden. ${ }^{3,4}$ Understanding the rates and drivers for hospitalizations and other acute episodes of care is important when considering treatment choices, costs, later lines of therapy, and clinical course.

Symptoms related to CRC chemotherapy treatment are the primary reason for unexpected hospitalizations, 5,6 although rates vary among previous studies. Hospitalization rates among younger patients (mean age 65 years) with any stage of CRC ranged from $12 \%$ to $53 \%$. ${ }^{7,8}$ In older patients (age 70 years or older) with metastatic CRC (mCRC), hospitalization rates ranged from $22 \%$ in the first 30 days to $44 \%$ in the first 4 months following chemotherapy. ${ }^{9-11}$ Jang et al reported $44 \%$ of patients with mCRC experienced a hospitalization for a serious adverse event. ${ }^{12}$ The highest overall rate of hospitalizations (78\%) was reported for an elderly population with early-stage CRC. ${ }^{13}$

Previous research on chemotherapy-related hospitalization among patients with CRC as well as other types of advanced cancer has also examined predictors of unplanned hospitalizations in specific populations, such as patients with certain types of cancer, in a particular age group, or treated at specific facilities. ${ }^{6-8,13-16}$ These studies found a higher risk of hospitalization among patients receiving 2 or more chemotherapy agents, women, those with comorbid conditions, and those with certain socioeconomic markers, such as non-White race, residence in a rural area, and low income. Additionally, researchers demonstrated patients in real-world settings had higher hospitalization rates than those reported in clinical trials, ${ }^{14,17}$ and they noted the need for further research on the real-world experience of patients receiving chemotherapy. ${ }^{17}$

To address this research need, our study examined patients with stage IV mCRC undergoing first-line chemotherapy from a large commercial health insurer in the US. Our objective was to characterize the frequency of and possible reasons for the occurrence of hospitalizations in this population.

\section{Methods}

This retrospective cohort study identified adults with mCRC using claims and clinical care data from the HealthCore Integrated Research Environment (HIRE). HIRE contains medical and pharmacy claims for 14 commercial health insurance plans across the United States. Researchers accessed data in the format of a limited data set for which data use agreements were in place with the covered entities in compliance with the Health Insurance Portability and Accountability Act (HIPAA) Privacy Rule.

We included patients with mCRC initiating treatment with a regimen that contained at least one of the five most frequently used first-line treatments for CRC (fluorouracil [5-FU], oxaliplatin, bevacizumab, irinotecan, and capecitabine) as observed in the database between June 23, 2014 and June 30, 2016, with the first claim set as the index date. All patients had $\geq 6$ months of health insurance plan enrollment prior to index date (baseline period).

The primary endpoint was all-cause hospitalizations, including both inpatient stays and stand-alone emergency department (ED) visits without inpatient admission, occurring up to 30 days after the end of first-line chemotherapy. We analyzed the sample in aggregate as well as stratified by chemotherapy agents in the form of five overlapping cohorts. Baseline characteristics were described as absolute/relative frequencies for categorical variables, and means, standard deviations [SD], and/or medians with interquartile ranges (IQR) for continuous variables. We compared characteristics between patients with and without hospitalizations using $\chi^{2}$ - and t-tests (univariate analysis). No statistical correction for multiple hypothesis testing was applied. The most common medical issues associated with inpatient hospitalizations were identified 
from the International Classification of Diseases, Ninth/ Tenth Revision, Clinical Modification (ICD-9/10-CM) codes found in the primary diagnoses fields in the medical claims during the stay. Logistic regression was used for the multivariate evaluation determining baseline patient factors associated with the occurrence of $\geq 1$ hospitalization.

\section{Results}

\section{Baseline Patient Characteristics}

A total of 717 patients were included in the study (285 hospitalized, 432 non-hospitalized) with a median followup time of 116 days (IQR 73-186). There were 34 distinct regimens used in the first-line setting (Appendix Table 1). Mean age was 55.2 years and $58 \%$ were male (Table 1). Eastern Oncology Cooperative Group (ECOG) performance status was most frequently $0(44 \%)$ or 1 (39\%). Approximately $25 \%$ of patients had undergone surgery in the 6 months prior to receiving first-line chemotherapy.

The most common first-line chemotherapeutic agents were 5 -FU ( $79 \%$ of patients with at least one claim on the index date), oxaliplatin (67\%), bevacizumab (58\%), irinotecan $(21 \%)$, and capecitabine $(19 \%)$. The majority of patients $(91 \%)$ received 2 or more agents. Liver was the most common site of metastasis $(51 \%)$, followed by lung $(22 \%)$. The most frequently reported comorbidities unrelated to cancer were mild liver disease $(43 \%)$, chronic pulmonary disease $(14 \%)$, and diabetes $(14 \%)$. Colon or rectal polyp was the most frequently reported cancerrelated comorbidity $(33 \%)$, followed by diverticulosis $(22 \%)$ and internal or external hemorrhoids (18\%).

\section{Hospitalizations During Follow-Up}

A total of 285 patients ( $40 \%$ ) had $\geq 1$ hospitalization, varying across treatment cohorts (40\% 5-FU, 38\% oxaliplatin, $42 \%$ bevacizumab, 49\% irinotecan, 39\% capecitabine; Figure 1). A small proportion (12\%) of patients had $\geq 2$ hospitalizations. Overall, 415 hospitalizations occurred, of which 260 were inpatient admissions and 155 were stand-alone ED visits. The mean time to the first hospitalization was 77 days (median 52, IQR 24-110), with a mean inpatient length of stay of 6 days (median 4, IQR 2-7). Infections/neutropenia (21\%), bowel-related complications $(17 \%)$, cardiac and circulatory disorders $(9 \%)$, malnutrition $(5 \%)$, pain $(5 \%)$, and renal disease $(2 \%)$ were identifiable issues associated with inpatient hospitalizations based on specific primary ICD diagnosis codes. In univariate analyses, insurance plan type, geographical location, higher ECOG scores, and renal disease were associated with hospitalization (Table 1).

Logistic regression showed no significant effect of most baseline patient demographic and clinical characteristics, as well as type of first-line agent used, on the occurrence of a hospitalization event (Figure 2). The only exception was an ECOG performance status of 1 or higher (OR 1.67; 95\% CI 1.20-2.34; $\mathrm{p}=0.003$ ).

\section{Discussion}

Our real-world data analysis found that approximately $40 \%$ of patients with $\mathrm{mCRC}$ were hospitalized while undergoing first-line chemotherapy. The type of chemotherapy agent (among the top 5 most frequently used in this database) did not affect the rate of hospitalizations with statistical significance. However, it is striking that more than 30 distinct regimens were used when there are far fewer evidencebased regimens for first-line chemotherapy. ${ }^{18}$ Hospital stays were typically short and associated with infections, neutropenia, or bowel-related complications.

These data provide another estimate for oncology practices regarding the expected hospitalization rates associated with the wide variety of first-line systemic therapies used to treat $\mathrm{mCRC}$. Our hospitalization rate of $40 \%$ is consistent with rates previously reported in younger patients with any stage of $\mathrm{CRC}^{7,12}$ as well as rates from studies in older patients with mCRC. 9,11 Hospitalization rates reported in clinical trials often underestimate the effects of chemotherapy when used in a realworld population. ${ }^{17}$ Clinical trials typically enroll patients with younger average age, fewer comorbid conditions, less organ damage (e.g. renal function), and better performance status than those seen in daily practice, and trials provide more intensive care and follow-up than the community setting.

The data reported in our real-world study may help reduce unnecessary variation in chemotherapy treatment patterns for $\mathrm{CRC}$, which are influenced by individual patient preferences and characteristics, and also by the treating physician. ${ }^{19}$ Treatment facilities may also use these data to develop anticipatory intervention plans to mitigate adverse events, ${ }^{20}$ support other quality improvement efforts, and prepare for alternative payment arrangements (for example, the Centers for Medicare and Medicaid Services is implementing value-based programs that provide incentive payments to physicians based on quality of service rather than quantity). ${ }^{21,22}$ 
Table I Baseline Patient Characteristics

\begin{tabular}{|c|c|c|c|c|}
\hline & Overall $(\mathrm{N}=7 \mid 7)$ & Hospitalized $(n=285)$ & Non-Hospitalized $(n=432)$ & P-value \\
\hline Follow-up (days), mean (SD) & $144.3(101.30)$ & $151.9(113.29)$ & $139.2(92.33)$ & 0.320 \\
\hline Age on index date (years), mean (SD) & $55.2(9.21)$ & $55.6(8.72)$ & $54.9(9.5 I)$ & 0.426 \\
\hline Male, n (\%) & $412(57.5)$ & $155(54.4)$ & $257(59.5)$ & 0.176 \\
\hline \multicolumn{5}{|l|}{ Insurance Plan Type, n (\%) } \\
\hline PPO & $440(61.4)$ & $174(61.1)$ & $266(61.6)$ & 0.888 \\
\hline HMO & $155(21.6)$ & $52(18.2)$ & $103(23.8)$ & 0.075 \\
\hline CDHP & $122(17.0)$ & $59(20.7)$ & $63(14.6)$ & 0.033 \\
\hline \multicolumn{5}{|l|}{ Region of Patient Residence on Index Date, $\mathrm{n}(\%)^{\mathrm{a}}$} \\
\hline Northeast & $78(10.9)$ & $29(10.2)$ & $49(11.3)$ & 0.623 \\
\hline Midwest & $257(35.8)$ & $121(42.5)$ & $136(31.5)$ & $<0.01$ \\
\hline South & $197(27.5)$ & $65(22.8)$ & $132(30.6)$ & 0.023 \\
\hline West & $177(24.7)$ & $64(22.5)$ & $113(26.2)$ & 0.261 \\
\hline \multicolumn{5}{|l|}{ ECOG Performance Status, $\mathrm{n}(\%)^{\mathrm{a}}$} \\
\hline 0 & $313(43.7)$ & $107(37.5)$ & $206(47.7)$ & $<0.01$ \\
\hline I & $281(39.2)$ & $128(44.9)$ & $153(35.4)$ & 0.011 \\
\hline $2+$ & $40(5.6)$ & $26(9.2)$ & $14(3.2)$ & $<0.01$ \\
\hline \multicolumn{5}{|l|}{ Tumor Characteristics, $\mathrm{n}(\%)^{\mathrm{a}}$} \\
\hline KRAS genotype mutation & $134(18.7)$ & $53(18.6)$ & $81(18.8)$ & 0.959 \\
\hline KRAS genotype wildtype & $198(27.6)$ & $81(28.4)$ & $117(27.1)$ & 0.695 \\
\hline \multicolumn{5}{|l|}{ Cancer-Related Treatment, n (\%) } \\
\hline Surgery (total/partial colectomy) & $178(24.8)$ & $69(24.2)$ & $109(25.2)$ & 0.757 \\
\hline Radiation therapy & $77(10.7)$ & $28(9.8)$ & $49(11.3)$ & 0.521 \\
\hline \multicolumn{5}{|l|}{ Metastasis, n (\%) } \\
\hline Liver & $368(51.3)$ & $156(54.7)$ & $212(49.1)$ & 0.138 \\
\hline Lung & $160(22.3)$ & $72(25.3)$ & $88(20.4)$ & 0.124 \\
\hline Brain & $9(1.3)$ & $3(1.1)$ & $6(1.4)$ & $0.692^{\mathrm{b}}$ \\
\hline Kidney & $8(1.1)$ & $4(1.4)$ & $4(0.9)$ & $0.551^{\mathrm{b}}$ \\
\hline Other & $203(28.3)$ & $89(31.2)$ & $114(26.4)$ & 0.159 \\
\hline $\mathrm{QCl}$ using non-cancer comorbidities, mean (SD) & $1.1(1.18)$ & $1.2(1.27)$ & $1.0(1.12)$ & 0.078 \\
\hline Top 5 Comorbidity Components of the $\mathrm{QCl}, \mathrm{n}(\%)$ & & & & \\
\hline Mild liver disease & $310(43.2)$ & $130(45.6)$ & $180(41.7)$ & 0.296 \\
\hline Chronic pulmonary disease & $100(13.9)$ & $44(15.4)$ & $56(13.0)$ & 0.349 \\
\hline Diabetes without chronic complications & $99(13.8)$ & $38(13.3)$ & $61(14.1)$ & 0.765 \\
\hline Peripheral vascular disease & $40(5.6)$ & $14(4.9)$ & $26(6.0)$ & 0.528 \\
\hline Renal disease & $28(3.9)$ & $17(6.0)$ & II (2.5) & 0.021 \\
\hline \multicolumn{5}{|l|}{ Colon Cancer-Related Comorbidities } \\
\hline Colon or rectal polyp & $234(32.6)$ & $91(31.9)$ & $143(33.1)$ & 0.743 \\
\hline Diverticulosis & $154(2 \mid .5)$ & $55(19.3)$ & $99(22.9)$ & 0.248 \\
\hline Internal or external hemorrhoids & $126(17.6)$ & $55(19.3)$ & $71(16.4)$ & 0.324 \\
\hline IBD & $70(9.8)$ & $31(10.9)$ & $39(9.0)$ & 0.414 \\
\hline Angiodysplasia & $2(0.3)$ & $\mathrm{I}(0.4)$ & I $(0.2)$ & $0.767^{\mathrm{b}}$ \\
\hline
\end{tabular}

Notes: ${ }^{a}$ For the remainder of patients not listed in these rows, the corresponding information was missing or unknown. ${ }^{\mathrm{b}} \mathrm{p}$-Values from $\chi^{2}$-tests as per protocol; confirmed to be non-significant with Fisher's exact test.

Abbreviations: CDHP, consumer-driven health plan; ECOG, Eastern Cooperative Oncology Group; HMO, health maintenance organization; IBD, inflammatory bowel disease; KRAS, K-Ras protein; PPO, preferred provider organization; QCI, Quan-Charlson Comorbidity Index; SD, standard deviation.

Our findings may also be useful during informed consent discussions to quantify the risks of hospitalization from first-line chemotherapy. For example, in the practice setting patients with $\mathrm{ECOG}=0$ and $\mathrm{ECOG}=1$ are often grouped based on the assumption of broadly similar clinical profiles. Our study found that ECOG $\geq 1$ was associated with a $67 \%$ 


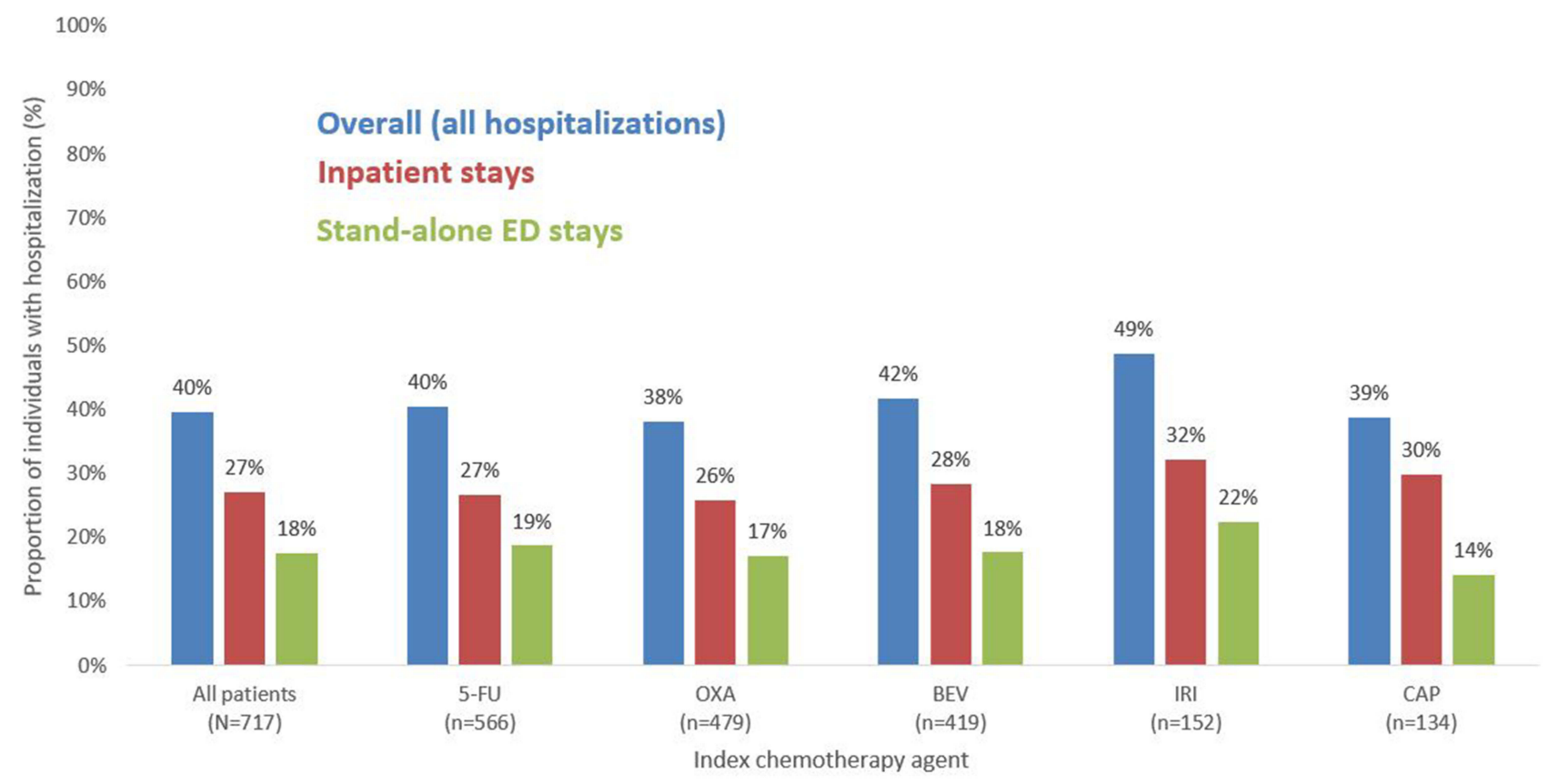

Figure I Frequency of hospitalization events (inpatient and/or ED).

\section{Baseline patient factors associated with hospitalization}

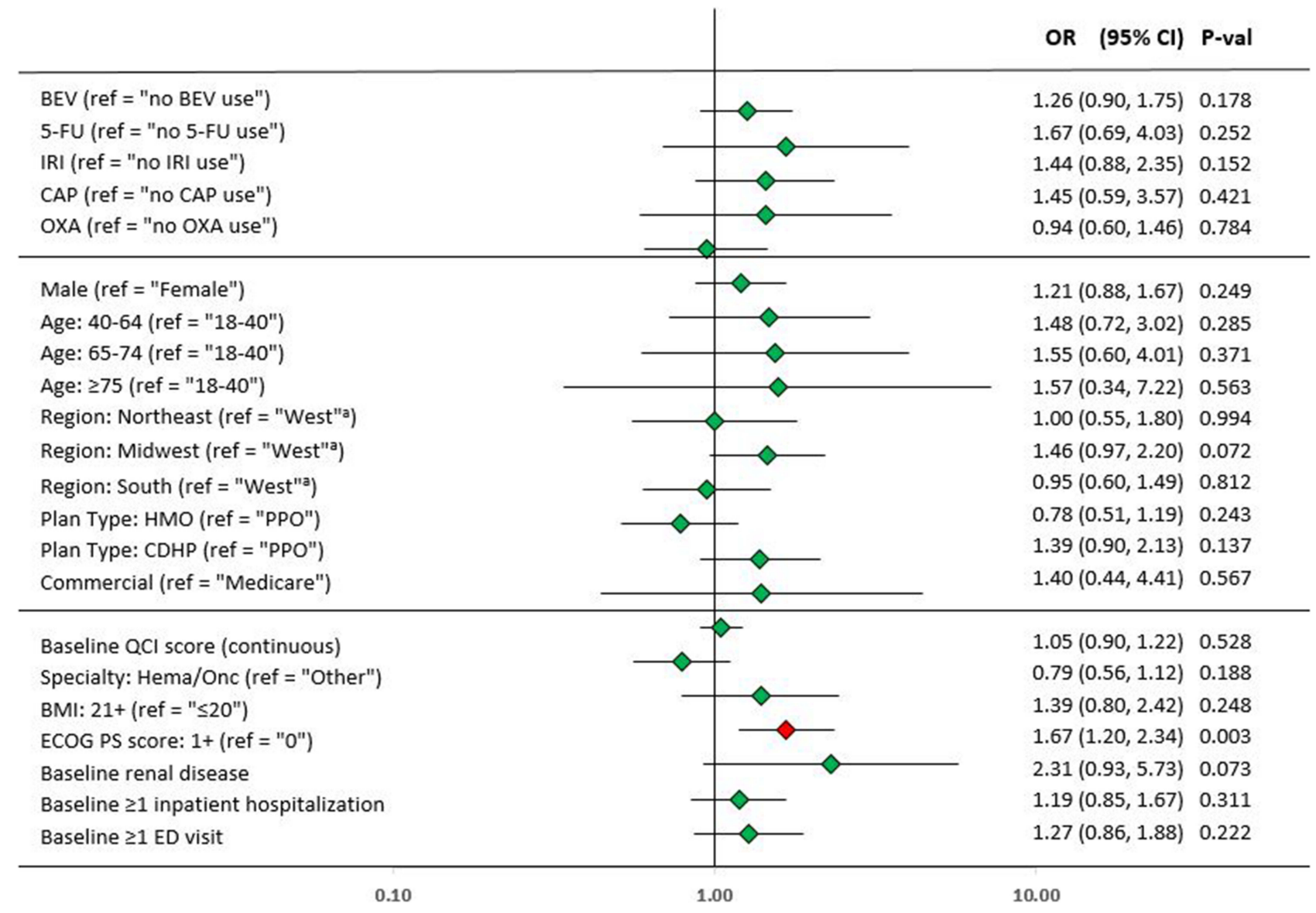

Figure 2 Logistic regression results.

Note: "Region reference group comprised of either "West" or "missing". 
increase in the odds of hospitalization compared with patients with $\mathrm{ECOG}=0$, suggesting that this part of the risk profile may actually diverge across the two groups. A previous study demonstrated improved patient outcomes - including reductions in ED visits and hospitalizations - when patients were engaged in monitoring their symptoms. ${ }^{20}$ Preparing patients more thoroughly for the effects of chemotherapy may therefore help improve outcomes.

\section{Limitations}

Due to sample size constraints with less frequently used medications, not all possible first-line chemotherapy agents could be analyzed. Follow-up time was limited and did not necessarily include the entire first-line therapy course. Treatment choices may have been confounded by observed and unobserved baseline factors, limiting the ability to compare outcomes across agents. The database did not contain information about whether hospitalizations were planned or unplanned or whether patients' treatment regimens were changed during or after the hospitalization. The impact of rare comorbidities (such as renal disease in this study) on the risks of hospitalization requires further research. Patients were identified from a large US claims database, limiting the generalizability of the findings to other countries or other US populations, such as those without insurance or those on Medicaid. As treatments become increasingly tailored to patients via targeted therapies, the applicability of these findings to patients with specific biomarkers will need to be examined.

\section{Conclusion}

A substantial proportion of patients with mCRC experienced a hospitalization while receiving chemotherapy. These data provide an initial estimate for oncology practices regarding the overall range of expected hospitalization rates associated with some of the currently used first-line systemic therapies for mCRC. Further research is needed to determine what proportion of these hospitalizations is potentially avoidable and what interventions can effectively reduce hospitalizations in patients with mCRC.

\section{Abbreviations}

CI, Confidence interval; CRC, Colorectal cancer; ECOG, Eastern Cooperative Oncology Group; ED, Emergency department; 5-FU, 5-fluorouracil; HIRE, HealthCore Integrated Research Environment; ICD-9/10-CM, International Classification of Diseases, Ninth/Tenth Revision, Clinical
Modification; IQR, Interquartile range; mCRC, Metastatic colorectal cancer; OR, Odds ratio; SD, Standard deviation.

\section{Ethics Approval and Consent to Participate}

Not required. HealthCore's federalwide assurance requires ethics review only for federally funded studies. Throughout this study, researchers' access was limited to data removed of all identifiers to ensure confidentiality. No study subjects were contacted and informed consent was not obtained. An Institutional Review Board did not review the study since only data in the format of a limited data set was accessed and HealthCore maintains data use agreements with the covered entities in compliance with the Health Insurance Portability and Accountability Act of 1996.

\section{Data Sharing Statement}

The data that support the findings of this study are available from HealthCore, but restrictions apply to the availability of these data to external sources, and therefore they are not publicly available. Data may be made available through the corresponding author upon reasonable request and with permission of HealthCore.

\section{Acknowledgments}

Cheryl Jones, an employee of HealthCore Inc. at the time of manuscript development, provided writing and editorial support for this manuscript. The results of this study have been previously presented in part at the 15th ASCO Gastrointestinal Cancers Symposium, San Francisco, CA, January 2018.

\section{Author Contributions}

All authors contributed to data analysis, drafting and revising the article, gave final approval of the version to be published, and agreed to be accountable for all aspects of the work.

\section{Funding}

Funding for this study was provided to HealthCore, Inc. by Eli Lilly and Company, Indianapolis, IN. The sponsor participated in the design and conduct of the study, data interpretation, and drafting and reviewing this manuscript.

\section{Disclosure}

Michael J Fisch is an employee of AIM Specialty Health, a subsidiary of Anthem, Inc. He also holds an appointment at the University of Texas MD Anderson Cancer Center. Michael Eleff is an employee of Anthem, Inc. Neither received funding 
for their participation in this study. Both are stockholders in Anthem, Inc. Michael Grabner, Joseph Singer, and John Barron are employees of HealthCore, Inc., which received funding from Eli Lilly and Company for the conduct of the study. All three are stockholders in Anthem, Inc. Amit D Raval and David M Kern were employees of HealthCore, Inc., when the study was conducted. Amit D Raval is now at Merck \& Co., Inc., Kenilworth, NJ, USA. David M Kern is now at Janssen Research \& Development, Titusville, NJ, USA. Lee Bowman, Collin Churchill, and Stewart Wetmore are employees and stockholders of Eli Lilly and Company. Daniel S Mytelka was an employee of Eli Lilly and Company when the study was conducted and is now at Massachusetts Institute of Technology, Cambridge, MA, USA. The authors report no other conflicts of interest in this work.

\section{References}

1. American Cancer Society. Key statistics for colorectal cancer. Available from: https://www.cancer.org/cancer/colon-rectal-cancer /about/key-statistics.html. Accessed June 5, 2019.

2. Centers for Disease Control and Prevention. Colorectal cancer statistics. Available from: https://www.cdc.gov/cancer/colorectal/statis tics/index.htm. Accessed June 5, 2019.

3. Calfee DP. Crisis in hospital-acquired, healthcare-associated infections. Annu Rev Med. 2012;63:359-371. doi:10.1146/annurevmed-081210-144458

4. Keefe DM, Elting LS, Nguyen HT, et al. Risk and outcomes of chemotherapy-induced diarrhea (CID) among patients with colorectal cancer receiving multi-cycle chemotherapy. Cancer Chemother Pharmacol. 2014;74(4):675-680. doi:10.1007/s00280-014-2526-5

5. Ang CW, Seretis C, Wanigasooriya K, Mahadik Y, Singh J, Chapman MA. The most frequent cause of 90-day unplanned hospital readmission following colorectal cancer resection is chemotherapy complications. Colorectal Dis. 2015;17(9):779-786. doi:10.1111/ codi.2015.17.issue-9

6. Gibson S, McConigley R. Unplanned oncology admissions within 14 days of non-surgical discharge: a retrospective study. Support Care Cancer. 2016;24(1):311-317. doi:10.1007/s00520-015-2786-6

7. Gonzales JR, Fernandez E, Moreno V, et al. Sex differences in hospital readmission among colorectal cancer patients. J Epidemiol Community Health. 2005;59:506-511. doi:10.1136/jech.2004.028902

8. Hassett MJ, Rao SR, Brozovic S, et al. Chemotherapy-related hospitalization among community cancer center patients. Oncologist. 2011;16:378-387. doi:10.1634/theoncologist.2010-0354

9. Delea T, Vera-Llonch M, Edelsberg JS, et al. The incidence and cost of hospitalization for 5-FU toxicity among medicare beneficiaries with metastatic colorectal cancer. Value Health. 2002;5(1):35-43. doi:10.1046/j.1524-4733.2002.51083.x

Cancer Management and Research

Publish your work in this journal

Cancer Management and Research is an international, peer-reviewed open access journal focusing on cancer research and the optimal use of preventative and integrated treatment interventions to achieve improved outcomes, enhanced survival and quality of life for the cancer patient.
10. Lai P, Sud S, Zhang T, Asmis T, Whealey-Price P. Palliative chemotherapy in advanced colorectal cancer patients 80 years of age and older. Curr Oncol. 2016;23(3):144-153.

11. Arapicio T, Jouve JL, Teillet L, et al. Geriatric factors predict chemotherapy feasibility: ancillary results of FFDC 2001-02 Phase III study in first-line chemotherapy for metastatic colorectal cancer in elderly patients. J Clin Oncol. 2013;31(11):1464-1470. doi:10.1200/ JCO.2012.42.9894

12. Jang RW-J, Enright K, Booth CM, Chan K, Yun L, Krzyanowska MK. Serious adverse events among a population-based cohort of patients receiving first-line chemotherapy for metastatic colorectal cancer (mCRC). J Clin Oncol. 2017;30(15).

13. Fessele KL, Hayat MJ, Mayer DK, Atkins RL. Factors associated with unplanned hospitalizations among patients with nonmetastatic colorectal cancers intended for treatment in the ambulatory setting. Nurs Res. 2016;65(4):24-34. doi:10.1097/NNR.0000000000000134

14. Du XL, Osborne C, Goodwin JS. Population-based assessment of hospitalizations for toxicity from chemotherapy in older women with breast cancer. J Clin Oncol. 2002;20(24):4636-4642. doi:10.1200/ JCO.2002.05.088

15. Nurgalieva Z, Liu CC, Du XL. Risk of hospitalizations associated with adverse effects of chemotherapy in a large community-based cohort of elderly women with ovarian cancer. Int J Gynecol Cancer. 2009;19(8):1314-1321. doi:10.1111/IGC.0b013e3181b7662d

16. Weaver C, Schiech L, Held-Warmkessel J, et al. Risk for unplanned hospital readmission of patients with cancer: results of a retrospective medical record review. Oncol Nurs Orum. 2006;33(3):E44-E52. doi:10.1188/06.ONF.E44-E52

17. Prince RM, Atenafu EG, Krzyanowska MK. Hospitalizations during systemic therapy for metastatic lung cancer: a systematic review of real world vs clinical trial outcomes. JAMA Oncol. 2015;1 (9):1333-1339. doi:10.1001/jamaoncol.2015.3440

18. Benson AB, Venook AP, Al-Hawary MM, et al. NCCN Clinical practice Guidelines in Oncology (NCCN guidelines ${ }^{\circledR}$ ). Rectal cancer, Version 1.2019. Available from: https://www.ncen.org/professionals/ physician_gls/pdf/rectal.pdf. Accessed June 5, 2019.

19. Abrams TA, Meyer G, Schrag D, Meyerhardt JA, Moloney J, Fuchs CS. Chemotherapy usage patterns in a US-wide cohort of patients with metastatic colorectal cancer. J Natl Cancer Inst. 2014;106(2):djt371. doi:10.1093/jnci/djt371

20. Basch E, Deal AM, Kris MG, et al. Symptom management with patient-reported outcomes during routine cancer treatment: a randomized controlled trial. J Clin Oncol. 2016;34(6):557-565. doi:10.1200/JCO.2015.63.0830

21. Centers for Medicare \& Medicaid Services. What are the value-based programs? Available from: https://www.cms.gov/Medicare/QualityInitiatives-Patient-Assessment-Instruments/Value-Based-Programs /Value-Based-Programs. Accessed December 4, 2019.

22. Centers for Medicare \& Medicaid Services. Value-based payment modifier and the physician feedback program. Available from: https://www. cms.gov/newsroom/fact-sheets/value-based-payment-modifier-andphysician-feedback-program. Accessed December 4, 2019.
The manuscript management system is completely online and includes a very quick and fair peer-review system, which is all easy to use. Visit http://www.dovepress.com/testimonials.php to read real quotes from published authors. 